\title{
Incidental Saccular Aneurysms on Head MR Angiography: 5 Years' Experience at a Single Large-Volume Center
}

\author{
Soonchan Park, ${ }^{\mathrm{a}, \mathrm{b}}$ Deok Hee Lee, ${ }^{\mathrm{a}}$ Chang-Woo Ryu, ${ }^{\mathrm{b}}$ Hae Wook Pyun, ${ }^{\mathrm{c}}$ Choong Gon Choi, ${ }^{\mathrm{a}}$ \\ Sang Joon Kim, ${ }^{a}$ Dae Chul Suh ${ }^{a}$ \\ ${ }^{a}$ Department of Radiology and Research Institute of Radiology, University of Ulsan College of Medicine, Asan Medical Center, Seoul, Korea \\ bepartment of Radiology, Kyung Hee University Hospital at Gangdong, School of Medicine, Kyung Hee University, Seoul, Korea \\ 'Department of Radiology, Pohang Stroke and Spine Hospital, Pohang, Korea
}

Background and Purpose The diagnostic accuracy for unruptured intracranial aneurysms has increased, and incidental asymptomatic aneurysms have come to represent a substantial clinical burden because of their controversial natural history. However, their prevalence may be attributable to variations in evaluation methods and demographics. We therefore describe the prevalence and magnetic resonance angiography (MRA) findings of incidental intracranial saccular aneurysms over a 5-year period at a single large-volume center.

Methods MRA images from 18,237 patients obtained between January 2001 and December 2005 were retrieved from the radiology report database. Patients diagnosed with incidental intracranial saccular aneurysms were identified and their MRA data were reviewed. Imaging and clinical follow-up data were evaluated.

Results During the study period, 366 incidental intracranial saccular aneurysms were identified in 330 patients (prevalence, 1.8\%; 95\% confidence interval, 1.63\%-2.01\%; 115 men and 215 women; age range, $22-82$ years; median age, 63 years). The prevalence was higher in women $(215 / 8,112)$ than in men $(115 / 10,125 ; P=0.02)$. The prevalence increased with age in women $(P<0.01)$, but not in men $(P=0.30)$. Aneurysm size ranged from $1.5 \mathrm{~mm}$ to 13 $\mathrm{mm}$, with a median size of $4 \mathrm{~mm}$. The most common location was the bifurcation of the middle cerebral artery $(131 / 366 ; 35.8 \%)$.

Conclusions Our real world experience indicated a slightly lower overall prevalence of incidental intracranial saccular aneurysms than previously reported. The prevalence increased with age in women but not in men.

Keywords MRI angiography; Aneurysm; Intracranial aneurysm

\author{
Correspondence: Deok Hee Lee \\ Department of Radiology, Asan Medical \\ Center, 88 Olympic-ro 43-gil, \\ Songpa-gu, Seoul 138-736, Korea \\ Tel: +82-2-3010-5944 \\ Fax: +82-2-476-0090 \\ E-mail:dhlee@amc.seoul.kr \\ Received: March 18, 2014 \\ Revised: May 2, 2014 \\ Accepted: May 21, 2014
}

The authors have no financial conflicts of interest.

\section{Introduction}

Unruptured intracranial aneurysms are not uncommon. The reported prevalence in the general population is $1 \%-9 \%$ in autopsy series ${ }^{1-4}$ and $0.5 \%-2 \%$ in imaging studies. ${ }^{4,5}$ This wide range in reported prevalence may be attributable to variations in eval- uation methods and demographics. The diagnostic accuracy for unruptured intracranial aneurysms has increased ${ }^{6}$ following the recent advent of non-invasive vascular imaging tools such as multi-channel computed tomography angiography and magnetic resonance angiography (MRA), and incidental asymptomatic aneurysms have come to represent a substantial clinical 
burden on account of their controversial natural history.

At our institution, a head MRA is performed for not only patients with ischemic stroke or a high number of cerebrovascular risk factors but also preoperative evaluation of patients with a planned major cardiac or transplantation surgery. Head MRA has also recently become a component of routine health checkups in selected cases when concerns regarding cerebrovascular disease are present. On several occasions, we were able to identify various cerebrovascular lesions such as steno-occlusive lesions. We further noted a gradual increase in the incidence of incidental asymptomatic aneurysms. Therefore, the aim of this study was to describe the incidental intracranial saccular aneurysms detected on MRA performed at our institution over a 5 -year period. We report the prevalence, anatomical location, size, and clinical follow-up findings of intracranial saccular aneurysms, as well as the gender and age of patients with incidental intracranial saccular aneurysms.

\section{Methods}

\section{Patient selection}

A search of the radiology report database identified 19,171 sets of MRA examinations from 18,237 patients that were obtained between January 2001 and December 2005. This period was selected so that at least 5 years of follow-up data could be available for all patients. All patients were ethnic Koreans. MRA was performed to detect various cerebrovascular diseases, to evaluate nonspecific cerebrovascular symptoms such as dizziness or dementia, as part of a standard health check-up, or as part of a preoperative evaluation in patients with planned major cardiac or transplantation surgery.

Patients with incidentally detected aneurysms were identified from the radiology reports (Figure 1). Patients with known intracranial aneurysms prior to the MRA examination and those with clear dissecting aneurysms or fusiform aneurysms were excluded. Patients with subarachnoid hemorrhage detected at the time of magnetic resonance (MR) imaging were also excluded. Patient data including age, gender, symptoms, clinical treatment course, and follow-up results were obtained from medical records.

\section{Acquisition of MR imaging sequences}

MR images were acquired on one of six 1.5-T MR scanners (2 Siemens Magnetom Avanto, Erlangen, Germany; 1 GE Signa CV/i, Milwaukee, USA; and 3 Philips Gyroscan Intera, Best, The Netherlands). A ramped radiofrequency pulse and threedirectional zero filling were used during data acquisition. MRA data were acquired using the three-dimensional time-of-flight
19,607 MRA evaluations performed between January 2001 and December 2005

436 excluded due to previous aneurysms, dissecting aneurysms, fusiform aneurysms, or SAH

19,171 MRA evaluations from 18,237 patients entered into the initial research

366 unruptured intracranial aneurysms identified in 330 patients

$$
\Rightarrow \begin{gathered}
43 \text { patients treated either by clipping } \\
(n=32) \text { or coiling }(n=11)
\end{gathered}
$$

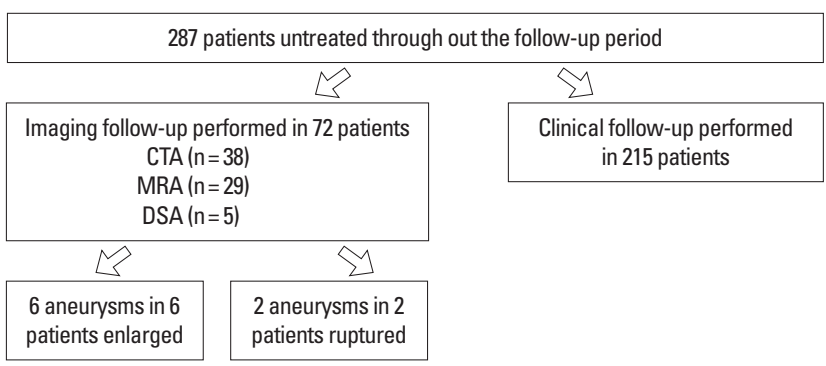

Figure 1. A flow chart summarizing the cases included in the study.

method focusing on the circle of Willis with the following parameters: echo time, $7 \mathrm{~ms}$; repetition time, $25 \mathrm{~ms}$; flip angle, $20^{\circ}$; one excitation; field of view, 160 or $200 \mathrm{~mm}$; matrix size, $256 \times 512$; voxel size, approximately $0.9 \times 0.9 \times 1.0 \mathrm{~mm}$; slab number, 4 . The first set of 12 maximum intensity projection images was generated by rotating around the sagittal axis at $15^{\circ}$. For the second and third sets of maximum intensity projection images, sub-volumes that contained the right and left circulation, respectively, were selected and 12 maximum intensity projection images were generated for each by rotating around the sagittal axis at $15^{\circ}$ to construct 24 images. In total, 36 images providing 12 stereoscopic images in three orthogonal directions were examined on the picture archiving and communication system for each patient. If more images were required due to the delicate delineation of the arterial structures, random image projections of volume rendering were created by reconstructing the source images using an Advantage Workstation (GE, Milwaukee, WI, USA).

\section{Image interpretation}

"Aneurysm" was used as a wild-card search word to identify the initial candidate cases of incidental aneurysms from among the 19,171 sets of MRA examinations contained in the radiology report database. Two experienced neuroradiologists (C-WR and HWP) used MRA images and medical reports to confirm the presence of an aneurysm and to quantify the size and location of the aneurysm. If there was a discrepancy between the 
original report and the reader's interpretation, or if the presence of an aneurysm was equivocal, another neuroradiologist (DHL) reviewed the records in order to reach a consensus. False-positive cases, in which the radiology report appeared in the initial search results but no aneurysm was found upon re-examination of the MRA images and medical records, were recorded and the cause of the false-positive was noted.

Aneurysm size was defined as the largest diagonal measurement seen on the maximum intensity projection images and classified as one of four categories modified from a previous study: ${ }^{7}<2 \mathrm{~mm}, 2-7 \mathrm{~mm}, 7-12 \mathrm{~mm}$, and 13-24 mm. Aneurysm location was described according to the secondary artery arising from the parent artery and classified as: paraclinoid internal carotid artery (ICA), distal ICA posterior wall, ICA bifurcation, anterior communicating artery, anterior cerebral artery, middle cerebral artery (MCA) trunk, MCA bifurcation, distal vertebral artery, basilar trunk, basilar top, posterior cerebral artery, superior cerebellar artery, or posterior inferior cerebellar artery. Paraclinoid ICA aneurysms included aneurysms originating near the anterior genu of the cavernous ICA or near the ophthalmic artery, and distal ICA posterior wall aneurysms included ICA aneurysms originating at the posterior communicating artery or the anterior choroidal artery. Multiplicity was checked and aneurysm numbers were recorded. In patients with multiple aneurysms, one index aneurysm with the largest size was selected for prevalence calculations. Patient age was categorized as 20-29 years, $30-39$ years, $40-59$ years, $60-79$ years, $70-79$ years, or $\geq 80$ years. Paired t-tests were performed to evaluate statistical differences in prevalence according to overall gender. Linear regression analyses were performed to test the relation between prevalence and age in men and women.

Table 1. The prevalence of incidental unruptured aneurysms according to age and gender

\begin{tabular}{|c|c|c|c|c|c|c|}
\hline \multirow[b]{2}{*}{ Age (yr) } & \multicolumn{3}{|c|}{ Men } & \multicolumn{3}{|c|}{ Women } \\
\hline & $\begin{array}{l}\text { Population } \\
\text { (n) }\end{array}$ & $\begin{array}{l}\text { Number } \\
\text { of IA(n) }\end{array}$ & $\begin{array}{c}\text { Preva- } \\
\text { lence }(\%)\end{array}$ & $\begin{array}{l}\text { Population } \\
\text { (n) }\end{array}$ & $\begin{array}{l}\text { Number } \\
\text { of IA (n) }\end{array}$ & $\begin{array}{c}\text { Preva- } \\
\text { lence (\%) }\end{array}$ \\
\hline $20-29$ & 134 & 1 & 0.75 & 142 & 1 & 0.70 \\
\hline 30-39 & 407 & 1 & 0.25 & 260 & 2 & 0.77 \\
\hline $40-49$ & 1,655 & 10 & 0.60 & 1,077 & 23 & 2.14 \\
\hline $50-59$ & 2,786 & 35 & 1.26 & 2,089 & 36 & 1.72 \\
\hline $60-69$ & 3,407 & 38 & 1.12 & 2,771 & 85 & 3.07 \\
\hline 70-79 & 1,444 & 28 & 1.94 & 1,514 & 57 & 3.76 \\
\hline$\geq 80$ & 292 & 2 & 0.68 & 259 & 11 & 4.25 \\
\hline Total & 10,125 & 115 & 1.14 & 8,112 & 215 & 2.65 \\
\hline
\end{tabular}

The prevalence was higher in women than in men $(P=0.02)$. The prevalence increased with age in women $(P<0.01)$ but not in men $(P=0.30)$.

IA, intracranial aneurysm.

\section{Results}

\section{Prevalence of incidental aneurysms}

Of the 18,237 patients studied, 330 ( 115 men and 215 women) had incidental aneurysms, resulting in a prevalence of $1.8 \%$ (95\% confidence interval, 1.6\%-2.0\%). The median age of patients with incidental aneurysms was 63 years and the age range was 22-82 years. Multiple aneurysms were found in 30 patients (9.1\%), including 26 patients with 2 aneurysms each, 3 patients with 3 aneurysms each, and 1 patient with 4 aneurysms. A total of 366 aneurysms were identified in 330 patients. Of these patients with incidental aneurysms, those assessed as part of a health check-up $(n=165)$ had more frequent aneurysms than patients with a previous stroke history $(n=136)$ or cardiac risk $(n=29)$.

The prevalence of incidental aneurysms was significantly higher in women $(215 / 8,112)$ than in men $(115 / 10,125 ; P=0.02$; Table 1; Figure 2) and nonsignificantly higher in men aged 70 79 years $(28 / 1,444)$ than in men aged $>80$ years $(2 / 292 ; P=$ 0.30 ; Table 1; Figure 2). Among women, the prevalence of aneurysms was highest in women older than 80 years in a linear regression analysis $(P<0.01)$. Prevalence increased with age in women $(P<0.01)$, but not in men $(P=0.30)$.

\section{Size and location of incidental aneurysms}

Aneurysm size ranged from $1.5 \mathrm{~mm}$ to $13 \mathrm{~mm}$, and the median size was $4.0 \mathrm{~mm}$. Most aneurysms (299/344; 86.9\%) were in the 2-7 mm category. Only two aneurysms measured $<2 \mathrm{~mm}$ $(0.6 \%)$.

The most common aneurysm location was the bifurcation of the MCA $(131 / 366,35.8 \%)$ and the next most common loca-



Figure 2. The relation between the prevalence of unruptured intracranial aneurysms and age group in men (diamonds) and women (circles). Prevalence increased with age in women $\left(R^{2}=0.932, P<0.01\right)$ but not in men $\left(R^{2}=0.1335\right.$, $P=0.30)$. 
Table 2. The location of incidental unruptured aneurysms

\begin{tabular}{lrrrc}
\hline & Men (n) & Women (n) & Total (n) & Rate (\%) \\
\hline Paraclinoid ICA & 13 & 38 & 51 & 13.9 \\
Distal ICA posterior wall & 5 & 30 & 35 & 9.6 \\
ICA bifurcation & 1 & 5 & 6 & 1.6 \\
Anterior communicating artery & 36 & 36 & 72 & 19.7 \\
Anterior cerebral artery & 5 & 9 & 14 & 3.8 \\
MCA trunk & 4 & 12 & 16 & 4.5 \\
MCA bifurcation & 44 & 87 & 131 & 35.8 \\
Distal vertebral artery & 1 & 0 & 1 & 0.3 \\
Basilar trunk & 3 & 0 & 3 & 0.8 \\
Basilar top & 7 & 15 & 22 & 6.0 \\
Posterior cerebral artery & 1 & 1 & 2 & 0.5 \\
Superior cerebellar artery & 0 & 11 & 11 & 3.0 \\
Posterior inferior cerebellar artery & 1 & 1 & 2 & 0.5 \\
Total & 121 & 245 & 366 & 100 \\
\hline
\end{tabular}

ICA, internal carotid artery; MCA, middle cerebral artery.

tion was the anterior communicating artery $(72 / 366,19.7 \%$; Table 2). Other frequent sites were the paraclinoid ICA (51/366, $13.9 \%)$, distal ICA posterior wall (35/366, 9.6\%), basilar top (22/366, 6.0\%), and MCA trunk (16/366, 4.5\%; Table 2). The bifurcation of the MCA was the most common location in both men and women (Table 2). There were 2 paraclinoid aneurysms in patients aged 20-29 years, and 2 MCA bifurcation aneurysms and 1 anterior communicating artery aneurysm in patients aged 30-39 years. In patients aged 40-49 years, the paraclinoid ICA was the most common site $(\mathrm{n}=10)$ followed by the anterior communicating artery $(n=7)$, and bifurcation of the MCA ( $\mathrm{n}$ =6). However, in patients aged 50-59 years, the MCA bifurcation was the most common site $(n=23)$ followed by the anterior communicating artery $(n=13)$ and paraclinoid ICA $(n=10)$. A similar tendency was observed in patients aged 60-69 years (MCA bifurcation, 49; anterior communicating artery, 22; paraclinoid ICA, 15) and those aged 70-79 years (MCA bifurcation, 34; anterior communicating artery, 21; paraclinoid ICA, 8). Among patients aged $\geq 80$ years, there were $3 \mathrm{MCA}$ bifurcation aneurysms, 3 distal ICA posterior wall aneurysms, and 3 basilar top aneurysms.

\section{False-positive cases}

Ninety false-positive cases, in which the radiology report contained the term "aneurysm" in the initial search results but no aneurysm was found on re-interpretation of the MRA images, were recorded. This high rate of false positives was probably due to cases that had 'rule out aneurysm' noted in the radiological report being included in the initial search results. In some of the cases, it was difficult to determine whether the lesion was an aneurysm even after a third neuroradiologist was consulted, mainly because of image resolution or image artifacts. The most common sites for false positives were the anterior communicating artery $(n=21)$ followed by the MCA bifurcation $(n=20)$ and the posterior communicating artery $(n=14)$. The causes of false positives were junctional dilatation $(\mathrm{n}=35)$, vascular ectasia $(n=24)$, and broad-based focal bulging $(n=14)$, which were all difficult to differentiate from MRA artifacts and vascular variations such as fenestration $(n=17)$. The study design precluded us from detecting false-negative cases.

\section{Follow-up results}

The duration of clinical follow-up ranged from 94 days to 2,641 days, and the median follow-up duration was 1,256 days, which was shorter than we intended. This was due to our inability to strictly follow up these patients. During the follow-up period, 43 of the 330 patients (13.0\%) were treated by clipping $(n=32)$ or coiling $(n=11)$. The treatment decision was made at various time points after the initial diagnosis of the aneurysm. Follow-up imaging was performed in 72 of the 287 untreated patients (25.1\%; computed tomography angiography in 38, MRA in 29, and digital subtraction angiography in 5). Follow-up imaging showed enlargement of the aneurysm in 6 of the 72 patients (8.3\%). Two of these were MCA bifurcation aneurysms, 2 were anterior communicating artery aneurysms, 1 was a distal ICA posterior wall aneurysm, and 1 was a basilar top aneurysm. Their initial size ranged from 4 to $6 \mathrm{~mm}$.

Two patients presented with a subarachnoid hemorrhage during follow-up. One was a 61-year-old female who presented with a mild headache. The initial MRA showed a 9-mm aneurysm at the anterior cerebral artery A2-3 junction. Subsequent computed tomography angiography showed a broad-neck aneurysm with a height of $4 \mathrm{~mm}$. The patient was reluctant to undergo surgery and was followed with imaging at regular intervals. Eight months after the initial diagnosis, the patient presented with a subarachnoid hemorrhage due to aneurysm rupture. The other patient was a 70-year-old female. The initial MRA was obtained to evaluate a brainstem infarction and showed a $3.5-\mathrm{mm}$ left MCA bifurcation aneurysm. Twenty-seven months after her initial diagnosis, while on antiplatelet therapy, the patient presented with a subarachnoid hemorrhage due to aneurysm rupture.

\section{Discussion}

With the advent of less invasive vascular imaging techniques, the incidental discovery of asymptomatic intracranial aneurysms is increasing. ${ }^{8}$ The clinical consequence and symptomatic conversion rate of these aneurysms is unknown and is a pressing clinical issue.

There are many reports on the prevalence of unruptured sac- 
cular intracranial aneurysms. ${ }^{4,5,9,10}$ However, the prevalence varies significantly across reports, probably due to the diversity of study methods and/or ethnic differences among study populations. One angiography-based study in a large cohort of patients reported that the prevalence of asymptomatic intracranial aneurysms was $0.65 \%,{ }^{9}$ and a recent meta-analysis reported a prevalence of $3.2 \%{ }^{4} \mathrm{~A}$ recent study evaluated the prevalence of unruptured intracranial aneurysms using 3-T MR imaging and reported a prevalence of $8.4 \%,{ }^{6}$ which is substantially higher than previous reports. ${ }^{4,5}$ This can be explained by an increased rate of detection of aneurysms smaller than $3 \mathrm{~mm}$ due to the higher resolution of the 3-T MR system. We found a prevalence of $1.8 \%$, which is somewhat lower than previous reports. ${ }^{410,11}$ There are several possible explanations for this difference. First, our study was not population-based and, although the patients were ethnically homogeneous, there may have been a bias in the study population. The lower rate might also be related to the low sensitivity of the 1.5-T MRA, a possibility supported by the small number of cases with aneurysms measuring less than $2 \mathrm{~mm}$. Another important possible explanation is the existence of falsenegative cases that were not included in the calculated prevalence. Because we identified incidental aneurysms based only on cases returned during our initial search of the radiology report database, there may have been a significant number of falsenegative cases that were not identified. In light of these limitations, we urge caution in interpreting our MRA prevalence rate. However, our study represents the real-world experience of a single high-volume center with uniform non-invasive imaging that we believe can contribute to the overall view of prevalence, distribution of aneurysms in terms of sites, and age.

Our results confirmed a higher prevalence of intracranial aneurysms in women than in men. ${ }^{10}$ The prevalence of aneurysms in women increased with age, which is a well-known risk factor. One study hypothesized that this might be associated with the decrease in estrogen concentration and estrogen-receptor density that occurs in women during and after menopause. ${ }^{12}$ The prevalence of aneurysms in men older than 80 years was lower than that in men aged 70-79 years, although the difference was not statistically significant $(P=0.30)$. Aneurysm prevalence was highest in women older than 80 years, and was higher in this group than in women aged 70-79 years. This is probably attributable to the relatively small sample of men aged over 80 years, which is due to the increased number of deaths in this age group.

A previous study demonstrated a gender difference in aneurysm location. ${ }^{13}$ However, there was no gender difference in aneurysm location in our study. Although some studies report that the paraclinoid ICA is the most common site for unruptured intracranial aneurysms, ${ }^{4,11,14}$ we found the most common site to be the MCA bifurcation, and this is consistent with other previous studies. ${ }^{15-18}$ Other frequent sites were the anterior communicating artery and the paraclinoid ICA. Before the age of 50, the most frequent aneurysm location was the paraclinoid ICA. However, the MCA bifurcation became the main predilection site after the age of 50. Significant increases in the prevalence of MCA bifurcation and anterior communicating artery aneurysms could be due to higher hemodynamic shear stress. ${ }^{19}$ Most aneurysms were between $2 \mathrm{~mm}$ and $7 \mathrm{~mm}$ in size. Aneurysms larger than $10 \mathrm{~mm}$ were rare, consistent with a previous report. ${ }^{9} \mathrm{An}$ eurysm enlargement on follow-up imaging was present in 6 cases, and aneurysm rupture during follow-up occurred in 2 cases. Enlargement or rupture therefore occurred in $2.8 \%$ of untreated patients. If all patients had been left untreated, the incidence of symptomatic conversion may have been higher than $2.8 \%$ as the decision to treat an aneurysm may have been based on the clinical perception of a high-risk lesion.

There were 90 false-positive cases that appeared in the initial database search. The causes of misclassification were junctional dilatation, vascular ectasia, broad-based focal bulging, and fenestration. False positives were most common in the anterior communicating artery. This is consistent with a recent report on the false-positive causes of aneurysms on MRA, ${ }^{20}$ and the causes of misclassification should be considered when analyzing MRA findings, especially for anterior communicating artery aneurysms. On the distal ICA, the differentiation of junctional dilatations from aneurysms may require additional image reconstruction. Many of the false positives in the current study could have been avoided with state-of-the-art MRA techniques, as many of the instances of vascular ectasia and bulging contours were a consequence of poor image quality.

Limitations inherent to the study design limit the conclusions that can be drawn from our results. The prevalence of incidental aneurysms may have been underestimated because we were unable to include false-negative cases, and the use of 1.5-T MRA data may have limited our ability to detect small aneurysms and thoroughly evaluate false-positive results due to limited resolution. In addition, the imaging follow-up data were limited because we could not strictly follow up these patients. We were therefore unable to obtain useful data on the natural history of incidental aneurysms.

\section{Conclusions}

We conducted a retrospective review of 18,237 patients who underwent MRA at our institution and found that the prevalence of unruptured intracranial aneurysms was $1.8 \%$, which was significantly higher in women than in men. The prevalence 
increased with age in women, but not in men. Before the age of 50 , the most frequent aneurysm location was the paraclinoid ICA. However, the MCA bifurcation became the main predilection site after the age of 50 . Therefore, overall, the most common location for unruptured intracranial aneurysms was the bifurcation of the MCA. There were a number of false-positive cases that appeared in our initial search, and the most common location for false-positive cases was the anterior communicating artery.

\section{References}

1. Jellinger K. Pathology of intracerebral hemorrhage. Zentralbl Neurochir 1977;38:29-42.

2. Stehbens WE. Aneurysms and Anatomical Variation of Cerebral Arteries. Arch Pathol 1963;75:45-64.

3. Inagawa T, Hirano A. Autopsy study of unruptured incidental intracranial aneurysms. Surg Neurol 1990;34:361-365.

4. Nakagawa T, Hashi K. The incidence and treatment of asymptomatic, unruptured cerebral aneurysms. J Neurosurg 1994;80: 217-223.

5. Winn HR, Jane JA, Sr., Taylor J, Kaiser D, Britz GW. Prevalence of asymptomatic incidental aneurysms: review of 4568 arteriograms. J Neurosurg 2002;96:43-49.

6. Igase K, Matsubara I, Igase M, Miyazaki H, Sadamoto K. Initial experience in evaluating the prevalence of unruptured intracranial aneurysms detected on 3-tesla MRI. Cerebrovasc Dis 2012; 33:348-353.

7. Wiebers DO, Whisnant JP, Huston J, 3rd, Meissner I, Brown RD, Jr., Piepgras DG, et al. Unruptured intracranial aneurysms: natural history, clinical outcome, and risks of surgical and endovascular treatment. Lancet 2003;362:103-110.

8. Yoon W. Current update on the randomized controlled trials of intracranial aneurysms. Neurointervention 2011;6:1-5.

9. Gibbs GF, Huston J, 3rd, Bernstein MA, Riederer SJ, Brown $\mathrm{RD}$, Jr. Improved image quality of intracranial aneurysms: 3.0T versus 1.5-T time-of-flight MR angiography. AJNR Am J Neuroradiol 2004;25:84-87.

10. Rinkel GJ, Djibuti M, Algra A, van Gijn J. Prevalence and risk of rupture of intracranial aneurysms: a systematic review. Stroke 1998;29:251-256.

11. Jeon TY, Jeon P, Kim KH. Prevalence of unruptured intracranial aneurysm on MR angiography. Korean J Radiol 2011;12:
$547-553$.

12. Harrod CG, Batjer HH, Bendok BR. Deficiencies in estrogenmediated regulation of cerebrovascular homeostasis may contribute to an increased risk of cerebral aneurysm pathogenesis and rupture in menopausal and postmenopausal women. Med Hypotheses 2006;66:736-756.

13. Horikoshi T, Akiyama I, Yamagata Z, Nukui H. Retrospective analysis of the prevalence of asymptomatic cerebral aneurysm in 4518 patients undergoing magnetic resonance angiography-when does cerebral aneurysm develop? Neurol Med Chir (Tokyo) 2002;42:105-112; discussion 113.

14. Burns JD, Huston J, 3rd, Layton KF, Piepgras DG, Brown RD, Jr. Intracranial aneurysm enlargement on serial magnetic resonance angiography: frequency and risk factors. Stroke 2009;40: 406-411.

15. Iwamoto H, Kiyohara Y, Fujishima M, Kato I, Nakayama K, Sueishi K, et al. Prevalence of intracranial saccular aneurysms in a Japanese community based on a consecutive autopsy series during a 30-year observation period. The Hisayama study. Stroke 1999;30:1390-1395.

16. Loumiotis I, Wagenbach A, Brown RD, Lanzino G. Small (\&lt; 10-mm) incidentally found intracranial aneurysms, Part 1: reasons for detection, demographics, location, and risk factors in 212 consecutive patients. Neurosurg Focus 2011;31:E3.

17. Huttunen T, von und zu Fraunberg M, Frosen J, Lehecka M, Tromp G, Helin K, et al. Saccular intracranial aneurysm disease: distribution of site, size, and age suggests different etiologies for aneurysm formation and rupture in 316 familial and 1454 sporadic eastern Finnish patients. Neurosurgery 2010;66:631638; discussion 638.

18. UCAS Japan Investigators, Morita A, Kirino T, Hashi K, Aoki $\mathrm{N}$, Fukuhara $\mathrm{S}$, et al. The natural course of unruptured cerebral aneurysms in a Japanese cohort. N Engl J Med 2012;366:24742482.

19. Meng H, Wang Z, Hoi Y, Gao L, Metaxa E, Swartz DD, et al. Complex hemodynamics at the apex of an arterial bifurcation induces vascular remodeling resembling cerebral aneurysm initiation. Stroke 2007;38:1924-1931.

20. Cho YD, Lee JY, Kwon BJ, Kang HS, Han MH. False-positive diagnosis of cerebral aneurysms using MR angiography: location, anatomic cause, and added value of source image data. Clin Radiol 2011;66:726-731. 